\title{
SAW Temperature Sensing for Automotive Test Bench Systems
}

\author{
Mag. Hannes Kurz, Dr. René Fachberger \\ sensideon $\mathrm{GmbH}$, Ligusterstraße 4, 4600 Wels
}

\begin{abstract}
:
Most measurement systems have evolved along with the evaluation and testing of piston engines and are currently being modified to meet the changing expectations with regards to electric engine testing. Hence, some such specific challenges require innovative and new testing and measurement solutions - e.g. Surface Acoustic Wave technology.
\end{abstract}

Key words: SAW, Heavy Duty Telemetry, engine temperature measurement, wireless sensing

\section{Problem}

The electrification of the automotive has raised much uncertainty in an entire industry and still, consumers debate about whether an electric engine can compete with the "good old" combustion engine when it comes down to convenience and performance.

Clearly, a comparison cannot easily be done but it emphasizes the high expectations towards eMobility. To improve the performance of such a powertrain system there are many variables that have to be taken into consideration. One of them is the temperature dynamic of the magnets inside the rotor. To keep track of this indicator, especially during driving operation, wired or optical measurement systems are unsatisfactory. Further, active metrology, especially one that builds up on amplifiers, usually shows limited resistance towards temperatures beyond $160^{\circ} \mathrm{C}$, electromagnetic influence, high velocities and oil abrasion. As a result, new approaches come into consideration and are being developed, to speed up and improve the development process of hybrid and full-electric drivetrains. One such approach is based on surface acoustic wave technology.

\section{Technology}

Surface Acoustic Wave (SAW), is already known to be suitable for remote temperature measurement under industrial conditions [1]. The sensors are robust, work passively and due to a batteryless function, the sensors can be applied under fairly high temperatures. However, a use in the field of automotive test bench development is rather unconventional.
The system configuration described in this paper is based on the principle, where the material specific change in resonance frequency [2], brought about by a temperature change, is being measured and converted into a temperature value.

\section{System Solution}

To represent sufficient added value, one main requirement was to create a design, suitable for both, vehicle and test bench testing and simultaneously to provide a reduced system, that affects the specimen to a minimum.

The applied telemetry system (Fig. 1) uses a modular design consisting of a transponder, a $2,4 \mathrm{GHz}$ antenna and the reader electronics. The transponder consists of a SAW resonator connected to a high temperature stable printed circiut board antenna. All components are tailored flexibly to meet the geometrical requirements of almost any engine design, no matter the rotor diameter, magnet type, rotational speed, oil cooling or electromagnetic interference.

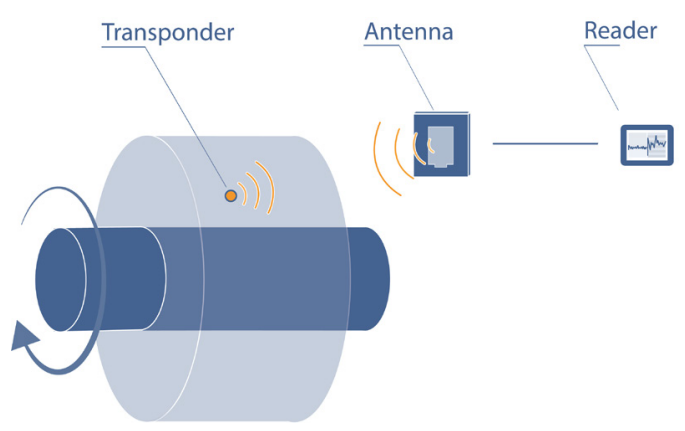

Fig. 1. System configuration SAW telemetry 


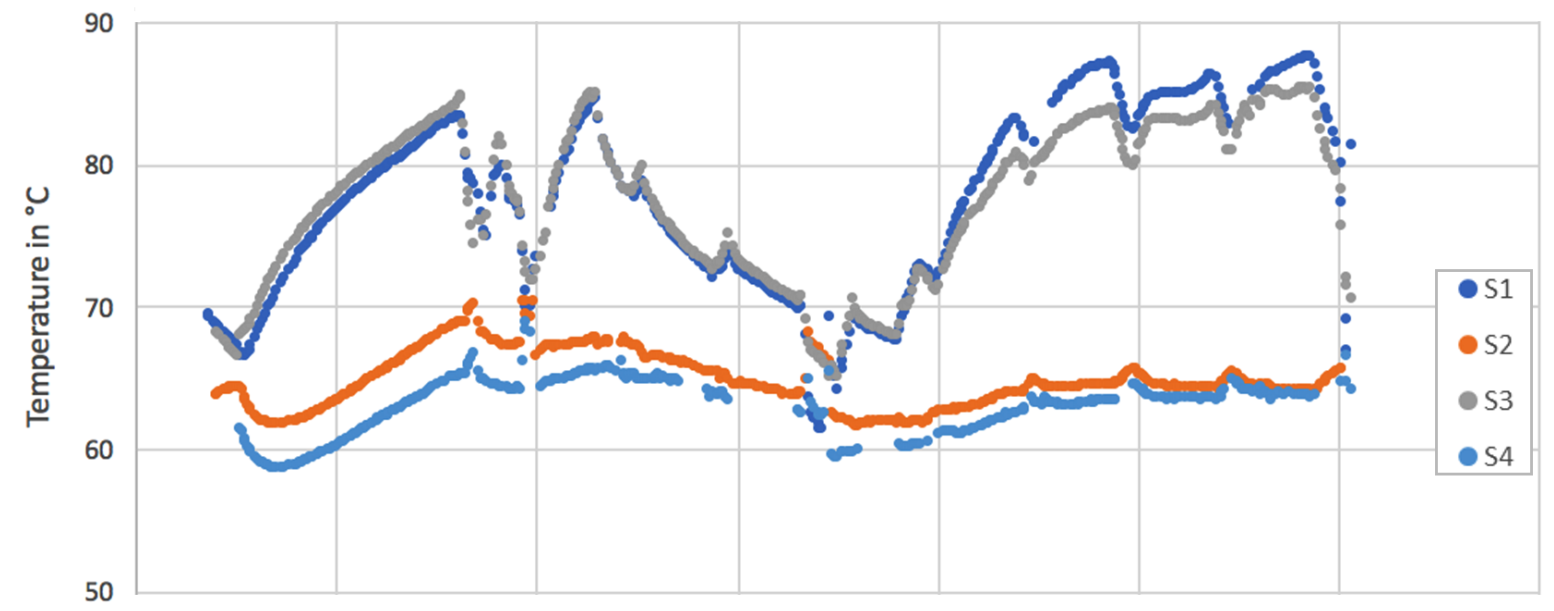

Fig. 2. Temperature plot over time (arb. unit) of a 4 channel SAW telemetry measurement with Stick Sensors S1 to S4 during vehicle prototype tests in Sweden

Due to the minimalistic design, the entire system gets mounted inside the engine housing without influencing oil jets or the performance.

\section{Test Bench vs. In-Car Measurement}

The environment of a test bench allows more flexibility when it comes to measurement systems. Cable routings may be more chaotic and it is possible to test single components of a powertrain separately. Telemetry systems can be relatively spacious since it is possible to mount them on shaft extensions for several reasons. When it comes to the testing of encapsulated modules or testing of integrated components especially during vehicle driving operation, to validate the entire system, shaft extensions and complicated wire routings become an issue.

The advantage of a SAW telemetry system is the ability to perform such measurements in any environment with no adaptions necessary. Fig. 2 shows an excerpt of vehicle measurements, recorded during driving performance tests in Sweden winter 2019. The specific challenge was to integrate a telemetry system into a prototype with no software based engine temperature management in order to show the performance potential of a new engine concept at an early stage in the means of a minimum viable product (MVP). Hybrid powertrains have even less available space when the engine is integrated into the gearbox.

\section{SAW Resonator Performance}

The measurement result shows a relatively high dynamic of the magnet temperature in certain driving modes. To capture this behavior, the telemetry is dependent on a sensor with an adequate sensor dynamic.
Fig. 3 displays the temperature step response of the stick sensor module used. After applying a temperature shock, $T_{90}$ is reached within 1.65 seconds, representing the time the sensor takes to reach $90 \%$ of the equilibrium value.

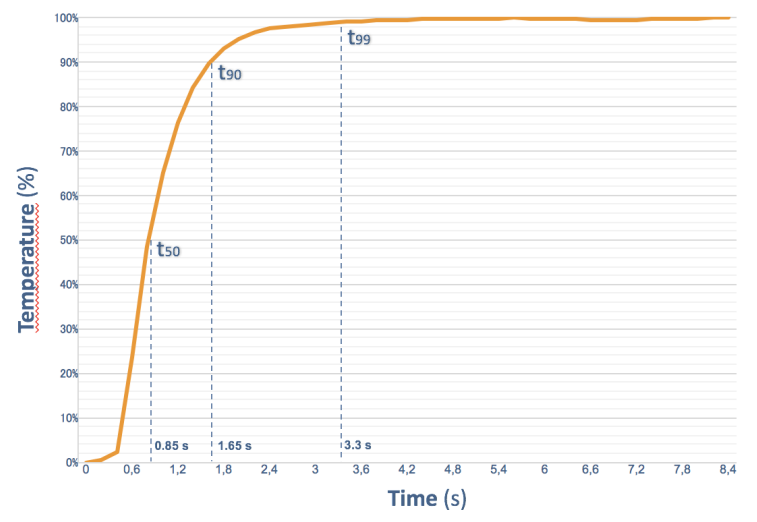

Fig. 3. Step response of Stick Sensor S

\section{Findings}

SAW temperature measurement systems are an enlargement of existing telemetry solutions. It addresses the demand to perform sophisticated measurement tasks in the field of automotive electric engine development.

The technology provides reliable and dynamical measurement data and performs such in places, that have not been accessible with conventional measurement technologies so far.

\section{References}

[1] R. Fachberger, C. Werner, "Wireless temperature monitoring in an electrolytic galvanizing plant," Proc. IEEE IUS, Prague, (2013)

[2] G. Bruckner, J. Bardong, "Wireless Readout of Multiple SAW Temperature Sensors," Sensors, 19, 3077, (2019) 